\title{
The AGAMOUS-LIKE 20 MADS domain protein integrates floral inductive pathways in Arabidopsis
}

\author{
Horim Lee, ${ }^{1}$ Sung-Suk Suh, ${ }^{1}$ Eunsook Park, ${ }^{1}$ Euna Cho, ${ }^{1}$ Ji Hoon Ahn, ${ }^{2}$ Sang-Gu Kim, ${ }^{1}$ \\ Jong Seob Lee, ${ }^{1}$ Young Myung Kwon, ${ }^{1}$ and Ilha Lee ${ }^{1,3}$ \\ ${ }^{1}$ School of Biological Sciences and Research Center for Cell Differentiation, Seoul National University, Seoul, 151-742, \\ Korea; ${ }^{2}$ Plant Biology Laboratory, The Salk Institute for Biological Studies, La Jolla, California 92037, USA
}

The very late-flowering behavior of Arabidopsis winter-annual ecotypes is conferred mainly by two genes, FRIGIDA (FRI) and FLOWERING LOCUS C (FLC). A MADS-domain gene, AGAMOUS-LIKE 20 (AGL20), was identified as a dominant $F R I$ suppressor in activation tagging mutagenesis. Overexpression of $A G L 20$ suppresses not only the late flowering of plants that have functional FRI and FLC alleles but also the delayed phase transitions during the vegetative stages of plant development. Interestingly, AGL20 expression is positively regulated not only by the redundant vernalization and autonomous pathways of flowering but also by the photoperiod pathway. Our results indicate that AGL20 is an important integrator of three pathways controlling flowering in Arabidopsis.

[Key Words: Flowering; MADS domain protein; AGL20; phase transition; activation tagging]

Received April 21, 2000; revised version accepted August 1, 2000.

Plants undergo several distinct phase transitions during their life cycle (Poethig 1990; Schultz and Haughn 1993; Telfer et al. 1997). The most dramatic phase change is the transition from vegetative growth to reproductive growth, or flowering. In addition, juvenile and adult phases can be distinguished during vegetative development, discernible by the distribution of trichomes on the leaf surface in Arabidopsis (Telfer et al. 1997). The transition to flowering is regulated by a complex genetic network that monitors the developmental state of the plant as well as environmental conditions. The genetic control of flowering has been extensively studied in Arabidopsis (Weigel 1995; Koornneef et al. 1998; Levy and Dean 1998). Arabidopsis is a facultative long-day plant that flowers faster under long days than short days. Genetic analyses of late-flowering mutants have identified more than 20 genes, which have been placed in at least three parallel genetic pathways based on the effect of each mutation on the response to environmental conditions. Genes such as CONSTANS (CO), GIGANTEA (GI), FT, $F W A, F H A, F E$, and $F D$ have been placed in the long day-dependent pathway because mutations in them cause lateness in flowering under long days but have little effect under short days. Genes such as FCA, FPA, FVE, FY, and LUMINIDEPENDENS (LD) have been

\footnotetext{
${ }^{3}$ Corresponding author.
}

E-MAIL ilhalee@plaza.snu.ac.kr; FAX 822-872-1993.

Article and publication are at www.genesdev.org/cgi/doi/10.1101/ $\operatorname{gad} .813600$. placed in the autonomous pathway because mutations in them cause delay in flowering under both long days and short days. A third pathway, which acts redundantly with the autonomous pathway, accelerates flowering upon vernalization (an extended cold treatment).

The genes defective in six late-flowering mutants, $L D$, $C O, F C A, F H A, G I$, and FT, have been isolated. $L D$ and $C O$ seem to encode transcription factors, whereas FCA encodes a putative RNA-binding protein that may have a role in posttranscriptional regulation (Lee et al. 1994a; Putterill et al. 1995; Macknight et al. 1997). FHA encodes a blue-light photoreceptor, CRYPTOCHROME 2, and GI encodes a protein that is regulated by a circadian clock (Guo et al. 1998; Fowler et al. 1999; Park et al. 1999|, suggesting that the two genes are involved in photoperiod perception. FT, whose biochemical function is unclear, acts downstream from CO (Kardailsky et al. 1999; Kobayashi et al. 1999).

Flowering-time genes modulate the activity of floral meristem identity genes such as $L E A F Y(L F Y)$ and APETALA 1 (AP1). The induction of $C O$ activity causes the rapid expression of $L F Y$ followed by later expression of AP1 (Simon et al. 1996). Conversely, FT and FWA function to activate $A P 1$ in parallel with $L F Y$ or downstream from LFY transcription (Ruiz-García et al. 1997). Genetic analyses have also shown that some genes such as CO, GI, FCA, and FVE affect the transcriptional induction of $L F Y$, whereas other genes such as FWA, FE, and $F T$ affect the response to $L F Y$ activity (Nilsson et al. 1998). Therefore, it has been suggested that flowering- 
time genes regulate flowering by either activating $L F Y$ expression or modulating the response to $L F Y$. Consistently, $L F Y$ expression is activated by the transition to flowering, and constitutive expression of LFY or AP1 causes early flowering (Mandel and Yanofsky 1995; Weigel and Nilsson 1995; Blázquez et al. 1997).

Genetic analyses of naturally-occurring variations of flowering time among Arabidopsis wild-type strains or ecotypes have identified additional loci that control flowering time. Among them, two loci, FRIGIDA (FRI) and FLOWERING LOCUS C (FLC), account for most of the difference in flowering time between early- and lateflowering ecotypes (Napp-Zinn 1985; Burn et al. 1993; Lee et al. 1993; Clarke and Dean 1994; Koornneef et al. 1994). Late-flowering ecotypes have dominant alleles of FRI and FLC that act synergistically to suppress flowering, whereas early-flowering ecotypes have a recessive allele of FRI and/or weak allele of FLC (Koornneef et al. 1994; Lee et al. 1994b; Sanda and Amasino 1995; Sheldon et al. 2000). FRI and FLC confer winter annual behavior to late-flowering ecotypes such that the effect of $F R I$ and FLC is suppressed by vernalization. FRI- and FLC-containing lines show delayed flowering under both long and short days, indicating that these two genes act in the autonomous pathway (Lee and Amasino 1995). FLC has been recently isolated and found to encode a MADSdomain protein (Michaels and Amasino 1999; Sheldon et al. 1999). Dominant, late-flowering alleles of FRI increase FLC transcript levels whereas vernalization decreases FLC levels. In addition, $L D$, a gene involved in the autonomous pathway, represses FLC expression. Therefore, it has been proposed that the modulation of FLC expression by the autonomous and vernalization pathways is critical to the control of flowering (Michaels and Amasino 1999; Sheldon et al. 2000).

To further elucidate the genetic control of flowering by FRI and FLC, we have screened for FRI suppressor mutants by activation-tagging mutagenesis. We have isolated a dominant FRI suppressor in which AGAMOUS-LIKE 20 (AGL20), a gene encoding a MADS-domain protein, is overexpressed. Our studies of AGL20 expression in various flowering-time mutants show that it is regulated by all three flowering-time pathways. Therefore, we propose that the level of AGL20 activity is critical to the control of flowering time and that AGL20 integrates signals from the photoperiod, vernalization, and autonomous floral induction pathways.

\section{Results}

\section{Isolation of an FRI suppressor by activation tagging}

To isolate FRI suppressor mutants, we performed activation-tagging mutagenesis (Hayashi et al. 1992; Weigel et al. 2000) in a line in which the FRI allele of the ecotype San Feliu-2 (SF2) was introgressed into Columbia (Col) through eight backcrosses (FRI-SF2; FLC-Col, referred to as FRI FLC below; Michaels and Amasino 1999). One of the primary transformants showed a very early-flowering phenotype and was designated as fsul-
1D/+ FRI FLC (fsul stands for FRI suppressor 1; Fig. 1A,B). T2 populations of $f s u 1-1 D /+F R I$ FLC showed an approximately 3:1 segregation ratio for the transgene marker (57 basta-resistant:20 basta-sensitive in basta selection; $\left.\chi^{2}=0.039, P<0.5\right)$, as well as for early to late flowering (56 early-flowering:24 late-flowering; $\chi^{2}=1.6$, $P<0.1)$. The late-flowering progeny produced more than 55 rosette leaves before bolting, which was similar to the FRI FLC parent. Early-flowering T2 plants fell into two classes. The very early class produced on average five rosettes, whereas the other class produced on average 11 rosette leaves. The ratio of the two classes was roughly $1: 2$ (16 very early plants:40 intermediately early plants, $\left.\chi^{2}=0.57, P<0.1\right)$. In addition, in the T3 population obtained from self-pollination of $\mathrm{T} 2$ progeny, all of the progeny from late-flowering plants were basta-sensitive (no T-DNA) and the progeny of the intermediately early class segregated 3:1 for basta resistance (hemizygous for T-DNA). The progeny of the very early class was uniformly basta-resistant (homozygous for T-DNA). The cosegregation of the T-DNA insert with the early-flowering phenotype and the simple 1:2:1 segregation ratio strongly indicated that a single T-DNA insertion was closely linked to the locus causing early flowering in fsu1-1D FRI FLC plants.

\section{Overexpression of AGL20 causes early flowering in FRI FLC}

DNA gel blot analysis confirmed that there was only a single T-DNA insert in fsu1-1D FRI FLC mutants (data
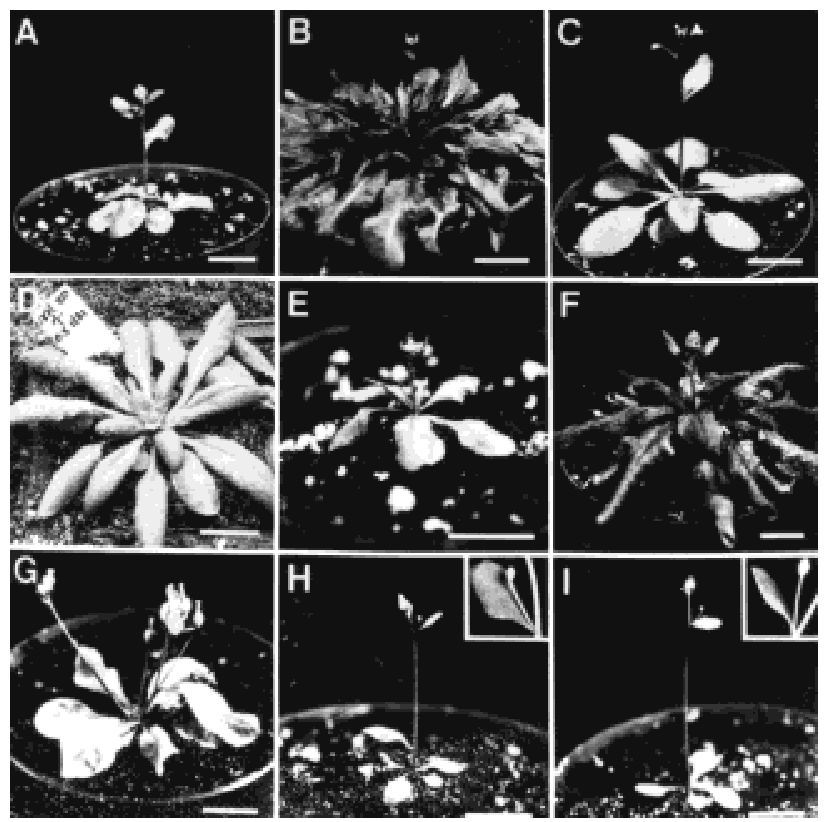

Figure 1. Flowering phenotype of lines described in this study. (A) Homozygous fsu1-1D FRI FLC; (B) FRI FLC; (C) Columbia; (D) ag120; (E) 35S::LFY/-; (F) 35S::LFY/- FRI/-; (G) fsu1-1D/35S::LFY/- FRI/-; (H) fsu1-1D FRI flc-3; (I) fsu1-1D fri-Col FLC. The insets in $(H)$ and $(I)$ show ectopic flowers subtended by cauline leaves. All plants were grown under long days $(16 \mathrm{~h}$ light/ 8 h dark). Bars, $1 \mathrm{~cm}$. 
not shown). Plant DNA flanking the right border of the T-DNA insertion site was isolated by plasmid rescue (Fig. 2A)(Lee et al. 1994a). Sequence analysis of rescued plant DNA revealed that the insertion was in a part of the genome represented by bacterial artificial chromosome (BAC) clone F17K2 (GenBank accession no. AC003680). The rescued plant sequences spanned nucleotides 63,367 to 64,214 of BAC F17K2 and included sequences of the first exon of AGL2O (Fig. 2B). The four

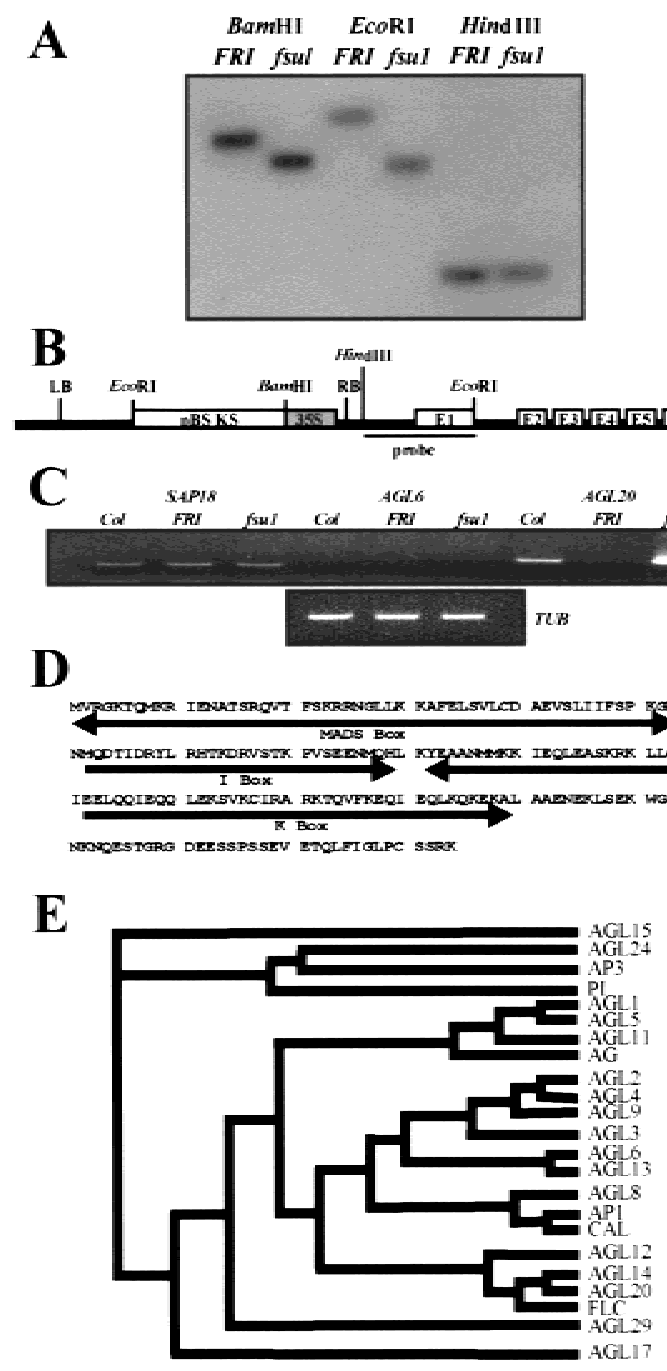

Figure 2. Insertion of $35 \mathrm{~S}$ enhancer in the promoter region of AGL20 causes overexpression. (A) DNA gel blot analysis showing the polymorphism between fsu1-1D FRI FLC (fsu1) and FRI FLC (FRI). (B) Diagram of genomic organization showing the

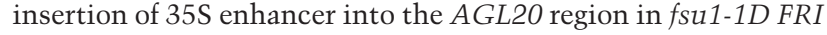
FLC. LB and RB are left border and right border of T-DNA, respectively. A bar represents the DNA fragment obtained by plasmid rescue and used for DNA gel blot analyses. (C) RT-PCR result for SAP18, AGL6, and AGL20 in the leaves of 3-week-old Columbia (Col), FRI FLC (FRI), and fsu1-1D FRI FLC (fsu1). The $\beta$-tubulin gene (TUB) was amplified as a quantitative control. (D) The amino acid sequence of AGL20. MADS, I, and K domains are indicated. (E) Neighbor-joining phylogenetic tree for Arabidopsis MADS-domain proteins. repeats of the $35 \mathrm{~S}$ enhancer were inserted 677 base pairs upstream of the AGL20 start codon in the fsu1-1D FRI FLC mutant.

Reverse transcription-polymerase chain reaction (RTPCR) of RNA isolated from the leaves of 3-week-old Columbia, FRI FLC, and the fsu1-1D FRI FLC mutant showed that AGL20 is overexpressed in fsu1-1D FRI FLC (Fig. 2C). Conversely, two putative genes near the TDNA insertion site, AGL6 (F17K2.18) and sin3 associated polypeptide (SAP18, F17K2.17) were not overexpressed in the fsu1-1D FRI FLC mutant (Fig. 2C). This result indicates that the acceleration of flowering in fsu1-1D FRI FLC results from overexpression of AGL20. Consistent with this, when the AGL20 coding sequence under control of the CaMV 35 S promoter was introduced into FRI FLC, all transgenic lines flowered earlier than FRI FLC (Table 1). Henceforth, we renamed fsu1-1D to ag120-101D.

AGL20 consists of seven exons and six introns (Fig. 2B). It encodes a typical MADS-domain protein that contains MADS, I (intervening), K, and C-terminal domains (Fig. 2D). Phylogenetic analysis using the M-I-K region showed that AGL20 is most similar to the MADS-domain genes AGL14 and FLC in Arabidopsis (Fig. 2E). The amino acid sequence identity in the $\mathrm{M}-\mathrm{I}-\mathrm{K}$ region is $65.4 \%$ with AGL14 and $43.6 \%$ with FLC, whereas overall amino acid sequence identity is $55.9 \%$ with AGL14 and $37.7 \%$ with FLC.

The normal role of AGL20 in the regulation of flowering was confirmed with a T-DNA insertional mutant, which flowered late in both long and short days (Fig. 1D). The ag120 mutants flowered later under short days than under long days, which indicates that the agl20 mutant is responsive to photoperiod (Table 1).

\section{ag120-101D FRI FLC responds to photoperiod and vernalization}

The effect of photoperiod and vernalization on the flowering time of ag120-101D FRI FLC is shown in Figure 3. Similar to Columbia, ag120-101D FRI flowered more rapidly under long days than under short days. Vernalization reduced flowering time in ag120-101D FRI FLC, but the reduction in ag120-101D FRI FLC was higher than in Columbia. Therefore, ag120-101D FRI FLC responds to both photoperiod and vernalization, indicating that ag120-101D does not abolish the sensitivity to either photoperiod or vernalization in the FRI FLC background.

\section{ag120-101D accelerates phase transition in FRI FLC}

In Arabidopsis, a marker of phase change is the distribution of trichomes on the leaf surface (Telfer et al. 1997). The leaves produced during the juvenile phase have trichomes only on their adaxial side, whereas the leaves produced during adult phase possess trichomes on both adaxial and abaxial sides. The cauline leaves produced during the reproductive phase lack trichomes on 
Table 1. Comparison of phase transitions and flowering time in the plants with different combinations of transgenics and mutations

\begin{tabular}{|c|c|c|c|c|}
\hline Genotype & $\begin{array}{l}\text { Juvenile } \\
\text { leaves }^{\text {a }}\end{array}$ & $\begin{array}{c}\text { Total rosette } \\
\text { leaves }\end{array}$ & $\begin{array}{l}\text { Cauline } \\
\text { leaves }^{\mathrm{b}}\end{array}$ & $n$ \\
\hline & \multicolumn{4}{|c|}{ Experiment $1^{\mathrm{c}}$} \\
\hline Columbia $^{\mathrm{d}}$ & $4.8 \pm 0.4$ & $9.2 \pm 0.4$ & $2.5 \pm 0.5$ & 20 \\
\hline FRI FLC & $9.3 \pm 0.5$ & $61.0 \pm 1.4$ & $12.5 \pm 0.7$ & 10 \\
\hline agl20-101D FRI FLC $\mathrm{e}^{\mathrm{e}}$ & $3.3 \pm 0.5$ & $5.2 \pm 0.4$ & $5.3 \pm 0.9$ & 25 \\
\hline ag120-101D/- FRI FLC $C^{\mathrm{f}}$ & ND & $10.6 \pm 1.4$ & $5.4 \pm 0.5$ & 27 \\
\hline $35 S:: L F Y /-F R I /-F L C$ & ND & $16.7 \pm 1.9$ & $7.4 \pm 1.7^{\star}$ & 7 \\
\hline ag120-101D/- 35S::LFY/ - FRI/ - FLC & ND & $7.0 \pm 1.1$ & $4.5 \pm 1.2^{\star}$ & 10 \\
\hline ag120-101D fri FLC & ND & $2.0 \pm 0.4$ & $3.5 \pm 0.7^{\star}$ & 17 \\
\hline agl20-101D FRI flc-3 & ND & $2.0 \pm 0.3$ & $3.3 \pm 0.6^{\star}$ & 21 \\
\hline 35S::AGL20 FRI FLC S1 & ND & 14 & 6 & 1 \\
\hline 35S::AGL20 FRI FLC S2 & ND & 12 & 7 & 1 \\
\hline \multirow[t]{2}{*}{ 35S::AGL20 FRI FLC S3 } & ND & 17 & 8 & 1 \\
\hline & \multicolumn{4}{|c|}{ Experiment $2^{\mathrm{g}}$} \\
\hline Columbia, LD & ND & $11.9 \pm 0.3$ & $3.3 \pm 0.2$ & 30 \\
\hline ag120, LD & ND & $26.2 \pm 1.0$ & $5.0 \pm 0.6$ & 5 \\
\hline Columbia, SD & ND & $30.0 \pm 1.0$ & $9.0 \pm 1.0$ & 30 \\
\hline agl20, SD & ND & $68.8 \pm 4.9$ & $15.2 \pm 0.8$ & 5 \\
\hline
\end{tabular}

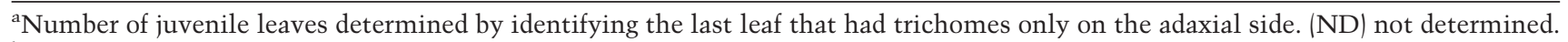
${ }^{\mathrm{b}}$ The cauline leaves produced in plants marked with an asterisk subtend ectopic flowers instead of secondary shoots.

${ }^{\mathrm{c}}$ In experiment 1, plants were grown under long days (16 h light $/ 8 \mathrm{~h}$ dark).

${ }^{\mathrm{d}}$ The genotype of Columbia is fri FLC.

'Homozygous ag120-101D FRI FLC.

${ }^{\mathrm{f}}$ Heterozygous ag120-101D/+ FRI FLC.

'In experiment 2, plants were grown under long days (LD) and short days (SD; $10 \mathrm{~h}$ light/14 h dark cycle).

their adaxial surface. To test whether ag120-101D also affected the transition from the juvenile to adult phase, we compared the trichome distribution in different lines (Table 1). FRI FLC produced approximately twice as many juvenile leaves as Columbia, indicating that FRI $F L C$ delays the transition from juvenile to adult phase as well as flowering. FRI FLC also produced more cauline leaves with associated secondary shoots than Columbia. The juvenile phase was found to be dramatically shortened in ag120-101D FRI FLC. However, ag120-101D FRI FLC produced significantly more cauline leaves than Columbia, although homozygous ag120-101D FRI FLC produced fewer rosette leaves than Columbia. This result suggests that $A G L 20$ overexpression can partially rescue the delay in the transition from secondary shoots to flowers in FRI FLC.

Interestingly, the flowering phenotype of ag120-101D FRI FLC is semidominant, if flowering time is measured by rosette leaf number (Table 1). That is, homozygous ag120-101D FRI FLC plants produce fewer rosette leaves than hemizygotes, indicating that the promotion of flowering by ag120-101D is dosage-dependent and that the effects of AGL20 overexpression are not saturated in ag120-101D FRI FLC. However, the number of cauline leaves with associated secondary shoots is not distinguishable between hemizygous and homozygous ag120-101D FRI FLC (Table 1). This result indicates differential effects of $a g 120-101 D$ on bolting and flowering.

\section{FRI FLC represses LFY expression}

An important regulator of the transition from secondary shoots to flowers is the $L F Y$ transcription factor (Weigel and Nilsson 1995). Therefore, we monitored $L F Y$ expression levels in ag120-101D FRI FLC using a LFY::GUS fusion gene (Blázquez et al. 1997). We compared $L F Y$ promoter activity in ag120-101D/+; FRI/fri; FLC/FLC, which flowered at the same time as ag120-101D/+ FRI FLC, with fri/fri; FLC/FLC (Columbia wild type). Hemizygous ag120-101D/+ FRI FLC produced five to six nodes of cauline leaves with secondary shoots above rosette leaves, but none of these showed GUS staining (Fig. 4A). Conversely, Columbia produced two to three cauline leaves with secondary shoots above rosette leaves, followed by flowers. In the Columbia background, GUS staining was detected in the cauline leaf primordia as well as floral meristems that are produced at the same nodes where secondary shoots are produced in hemizygous agl20101D FRI FLC plants (Fig. 4B). This result indicates that $L F Y$ expression is repressed by FRI FLC and that AGL20 overexpression cannot overcome such repression at least until after five to six secondary shoots are formed.

It has been reported that $35 S:: L F Y$ can partially rescue the late-flowering phenotype of FRI FLC (Nilsson et al. 1998). Reexamination of the effect of $35 S:: L F Y$ on FRI $F L C$ confirmed the previous report such that the number of rosette leaves was reduced dramatically by 35S::LFY. But the number of cauline leaves in FRI FLC was not 

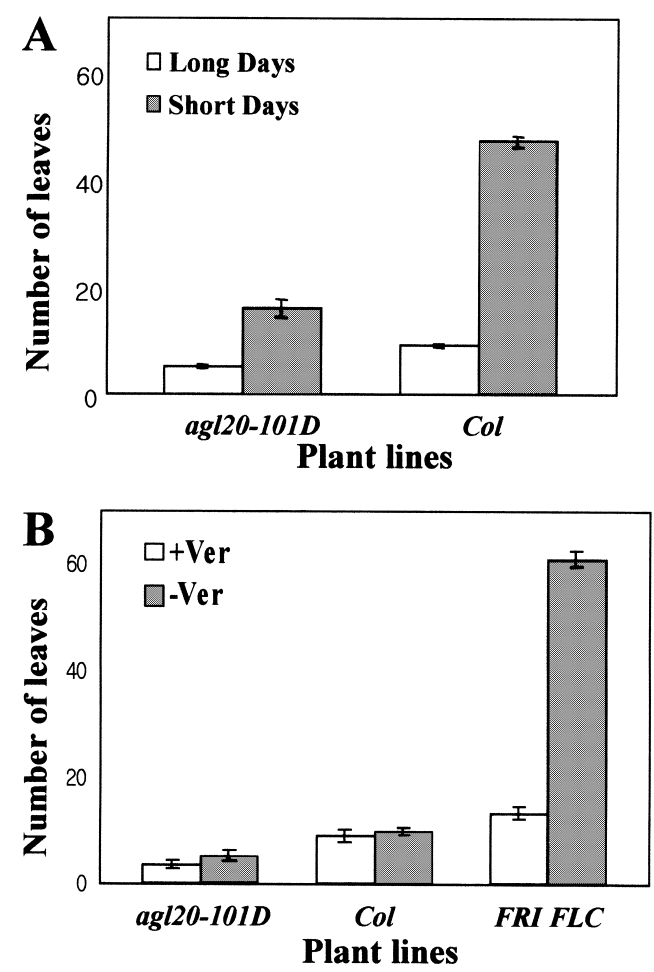

Figure 3. Effect of photoperiod and vernalization on the flowering time of ag120-101D FRI FLC. (A) The effect of photoperiod; $(B)$ the effect of vernalization. agl20-101D FRI FLC is designated as ag120-101D. Flowering time was measured as the number of rosette leaves. ag120-101D was originally designated fsu1-1D.

correspondingly reduced by 35 S::LFY (Fig. $1 \mathrm{~F}$; Table 1 ). However, all of the secondary shoots in the axils of cauline leaves were converted to solitary flowers by $35 S:: L F Y$. This result is in contrast to the effect of agl20$101 D$ on FRI FLC because secondary shoot-to-flower conversions were not observed in ag120-101D FRI, even though ag120-101D FRI FLC bolted earlier than 35S::LFY in the FRI FLC background (Table 1). This result may suggest that $A G L 20$ and $L F Y$ affect flowering through at least partially independent pathways. Consistent with this hypothesis, the effect of ag120-101D and 35S::LFY on flowering was additive such that both rosette leaf and cauline leaf numbers were reduced in ag120-101D/$35 S:: L F Y /$ - plants when compared with agl20-101D/- or $35 S:: L F Y /-$ in the FRI FLC background (Fig. 1G; Table 1).

Because FRI FLC delays both flowering time and the transition from cauline leaves with secondary shoots to flowers, we investigated the phenotype of ag120-101D in the absence of FRI or FLC. We combined the fri-Col allele, which is nonfunctional, with ag120-101D as well as the flc-3 null mutant allele (Levy and Dean 1998; Michaels and Amasino 1999). Flowering time was further accelerated by the addition of fri-Col or flc-3 mutations (Fig. 1; Table 1). The rosette leaf number was reduced to two in both agl20-101D FRI flc-3 and ag120-101D fri-Col $F L C$ lines. Interestingly, both lines showed solitary flowers in the axils of cauline leaves (Fig. 1H,I). Such a con- version of secondary shoots to flowers is also observed in 35S::LFY or 35S::AP1 plants (Mandel and Yanofsky 1995; Weigel and Nilsson 1995). This result may indicate that AGL20 overexpression can activate flower meristem identity genes such as $L F Y$ or $A P 1$ in shoot meristems, once FRI FLC is removed.

\section{Expression of AGL20}

The expression of AGL20 RNA was determined by in situ hybridization in Landsberg erecta (Ler) wild type (Fig. 4C,D). The strong expression of AGL20 in the shoot apical meristem and slightly weaker expression in the leaf primordia were detected during vegetative growth 18 days after germination). Four days later when shoot apical meristem produced flower meristems, the expression domain of AGL2O was expanded in the shoot apical meristem, and AGL2O transcript was also detected in developing leaves. To gain further insight into the role of AGL20 in regulating phase transition and flowering, we determined AGL20 expression levels in various tissues by RT-PCR (Fig. 5). In Columbia wild type, AGL20 was expressed most strongly in leaves, but the transcript was also detected in vegetative apices, inflorescence, stems of flowering plants, and roots (Fig. 5A). Temporal changes in AGL2O expression were also determined in whole plants. In our long-day conditions, flowers in Columbia wild type initiate around 9 days after germination, as determined by first detection of AP1 expression (Fig. 5B). AGL20 expression was increased significantly around 9 days after germination (Fig. 5B).

To investigate how AGL20 expression is regulated by $F R I$ and FLC, RT-PCR was performed using RNA extracted from whole plants of FRI FLC, FRI flc-3, and fri (FN235) FLC mutants at 6, 9, and 12 days after germination (Fig. 5C). AGL20 expression was very weak in FRI $F L C$, but an increased level of AGL2O expression was detected in fri (FN235) FLC or FRI flc-3 mutants. The expression level in fri (FN235) FLC or FRI flc-3 mutants was similar to that in Columbia. This result suggests that AGL20 expression is negatively regulated by FRI and FLC. Conversely, FLC expression level was increased in FRI FLC but decreased in the fri (FN235) FLC mutant, confirming that $F R I$ positively regulates $F L C$, as previously reported (Fig. 5C)(Michaels and Amasino 1999).

The effect of photoperiod on AGL20 expression was also determined. For this experiment, Columbia was grown under long and short days, and plants at a similar developmental stage (when the plants showed four rosette leaves; 9 days old under long days and 14 days old under short days) were compared for AGL20 expression level. Plants grown under short days showed approximately $40 \%$ less AGL20 expression than plants grown under long days, in contrast to $F L C$ expression, which showed no difference between long days and short days (Fig. 5D).

To determine the effect of vernalization on AGL20 expression, the FRI FLC line was used, because FRI FLC 

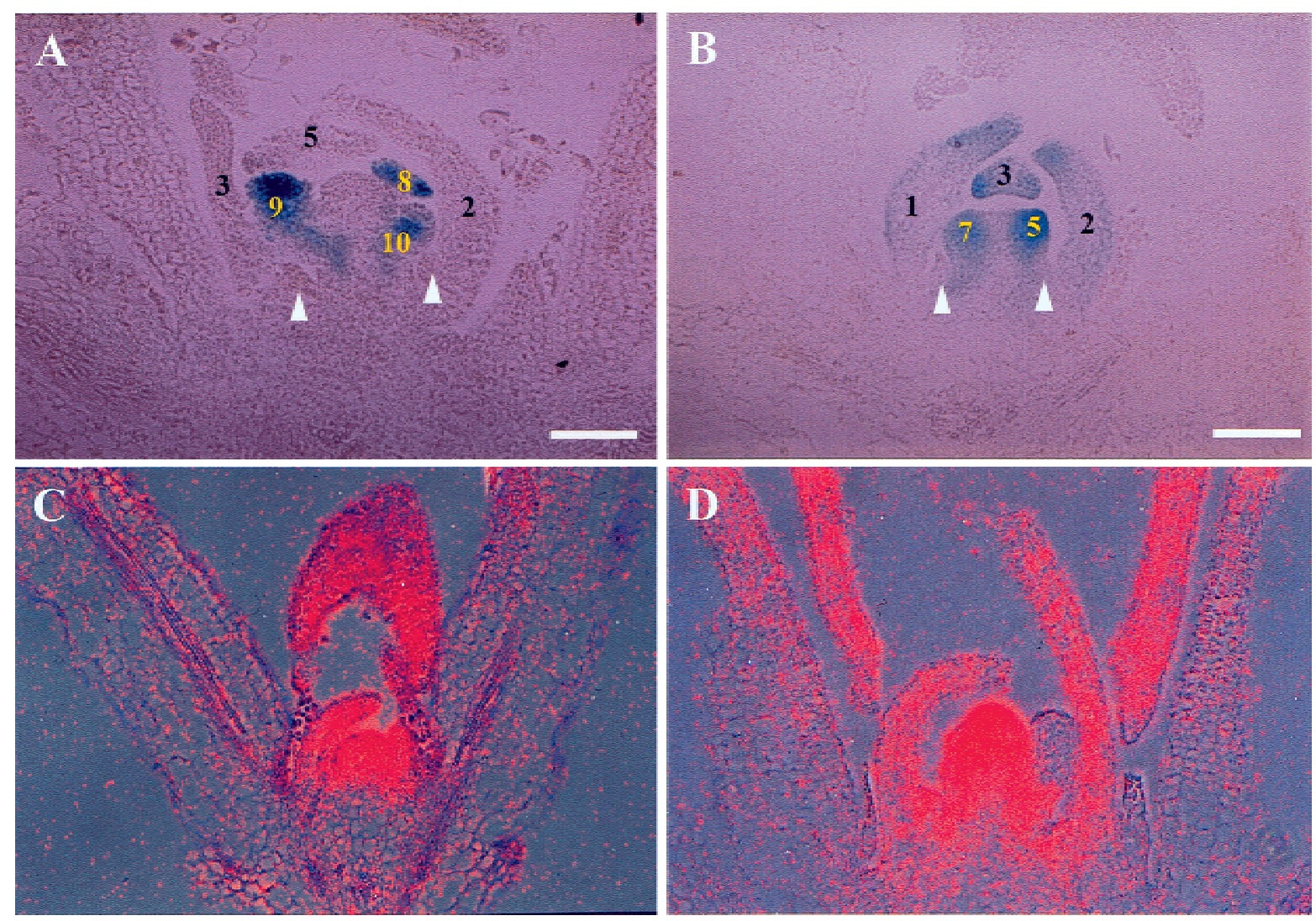

Figure 4. Analysis of LFY promoter activity in ag120-101D FRI FLC and in situ localization of AGL20 RNA in Ler. Expression of a LFY::GUS transgene introduced into ag120-101D FRI FLC (A) and Columbia $(B)$. The numbers on leaf primordia and floral meristems indicate node numbers above rosette leaves. Arrowheads indicate the position of secondary shoot primordia. In situ localization of $A G L 20$ in the shoot apices of 8-day-old $(C)$ and 12-day-old $(D)$ Ler plants. ag120-101D was originally designated fsu1-1D. Bars, 50 $\mu$ m.

shows strong acceleration of flowering by vernalization. The FRI FLC line was subjected to 4 weeks of cold treatment and was further grown under long days until it produced four rosette leaves. As a control, the FRI FLC line was grown under long days for 9 days, at which time it produced four rosette leaves, without vernalization. AGL20 was barely detectable by RT-PCR in the FRI FLC line without vernalization. However, 4 weeks of vernalization dramatically increased the AGL20 expression level (Fig. 5D). Conversely, vernalization reduced the expression level of $F L C$, consistent with previous reports (Fig. 5D)(Michaels and Amasino 1999). Thus, vernalization of the FRI FLC line causes acceleration of flowering and a concomitant decrease of FLC and increase of AGL2O expression. We also determined the AGL20 expression level in vernalized fca-1, which is in the Ler background. Although Ler has only a weakly active $F L C$ allele, flowering of $f c a-1$ is strongly accelerated by vernalization. When vernalized, AGL2O expression increased and FLC expression decreased in $f \mathrm{ca}-1$ (Fig. 5D). This suggests that vernalization, which represses FLC expression, activates AGL20. In conclusion, we found that AGL20 expression levels are regulated by both photoperiod and vernalization.
AGL20 expression is regulated by other flowering-time genes

We next investigated whether genes in the autonomous pathway regulate AGL20 expression. AGL20 expression was strongly reduced in the fve-3 mutant $(70 \%$ reduction compared with Columbia wild type), whereas $F L C$ was increased (Fig. 5F). Reduced AGL2O expression and increased FLC expression were also observed in the $1 d-1$ mutant (Fig. 5F). A simple model to account for this and the results presented above is that FLC is an upstream negative regulator of $A G L 20$, and that genes such as $L D$ or FVE activate $A G L 20$ expression through repression of $F L C$. Alternatively, AGL20 and FLC may repress each other's expression, and the increased FLC expression in $l d-1$ and fve-3 leads to the repression of AGL2O expression. To distinguish the two possibilities, we compared FLC expression level in agl20-101D FRI FLC, 35S::AGL20 FRI FLC, and FRI FLC (Fig. 5E). FLC expression levels in all of the lines were very similar, although higher expression of AGL20 was detected in ag120-101D and $35 S:: A G L 20$ in FRI FLC background. In addition, $F L C$ expression levels in agl20 loss-of-function mutants was similar to that of Columbia (Fig. 5E). These results show that AGL2O does not repress FLC expression and 
Lee et al.

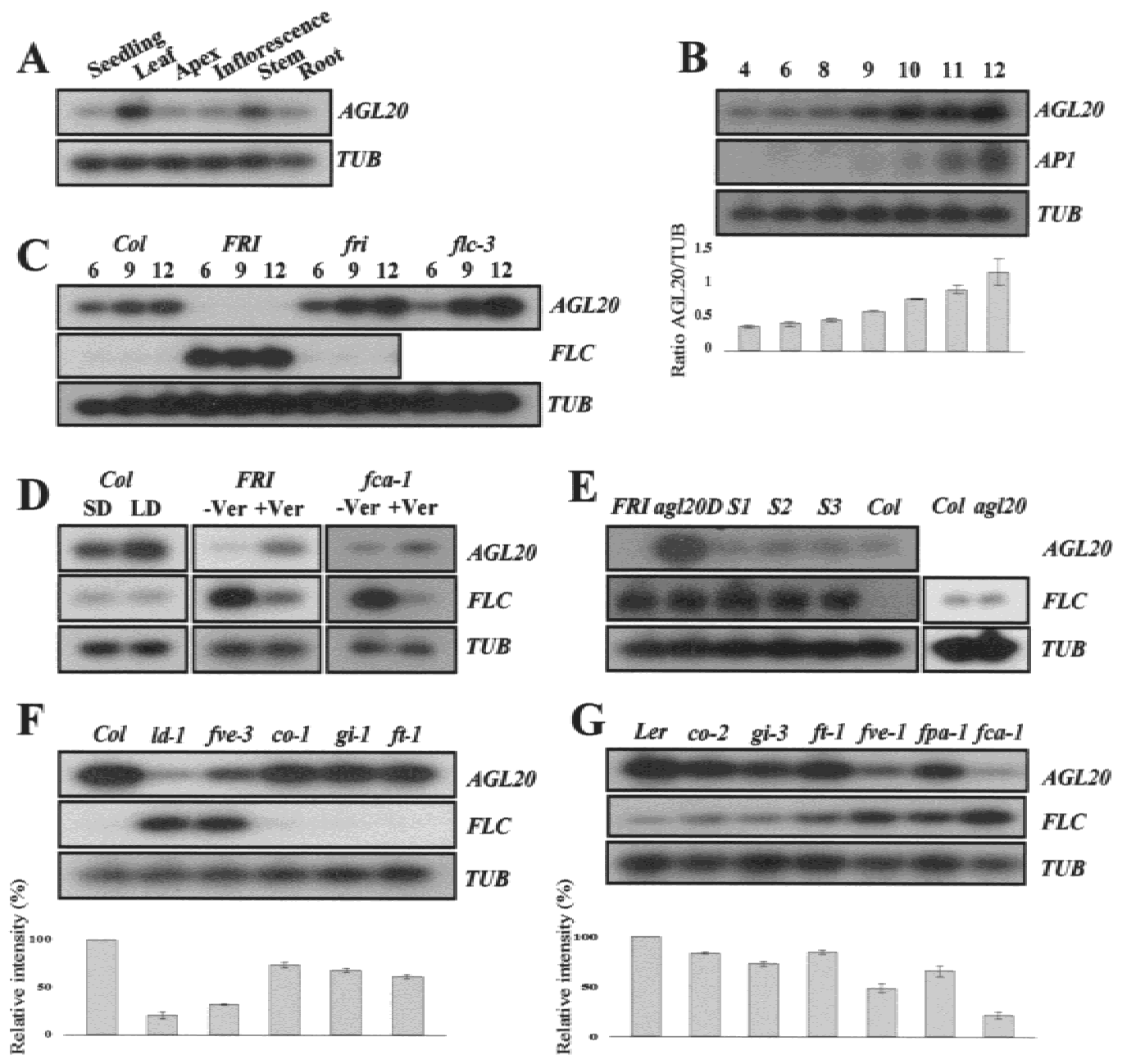

Figure 5. Analysis of AGL20 expression by RT-PCR. (A) Expression of AGL20 in various organs. (B) A time course of expression of $A G L 20, F L C$, and AP1. Lane numbers indicate days after germination. (C) Comparison of expression levels of AGL20 and FLC in Columbia (Col), FRI FLC (FRI), fri (FN235) FLC (fri), and FRI flc-3 (flc-3). (D) Effect of photoperiod and vernalization on the expression of AGL20 and FLC. To detect FLC expression in Columbia grown under short days and long days, the filter was overexposed. (E) Expression of AGL20 and FLC in ag120-101D FRI FLC (ag120D), 35S::AGL20 FRI FLC (S1, S2, S3), and ag120 knockout mutant. For ag120, the filter was overexposed to detect FLC. $(F)$ Expression of AGL20 in late-flowering mutants in Columbia background. (G) Expression of $A G L 20$ in late-flowering mutants in Ler background. The bar graphs below panels $B, F$, and $G$ represent the relative expression level of AGL20. Standard deviation obtained from three independent experiments is indicated. The $\beta$-tubulin gene (TUB) was amplified as a quantitative control.

support the idea that $F L C$ is an upstream negative regulator of $A G L 20$

We also compared how other late-flowering mutants affect the expression levels of AGL2O and FLC (Fig. $5 \mathrm{~F}, \mathrm{G})$. In the Columbia background, which has a dominant FLC allele, mutants of the photoperiod pathway, such as co-1, gi-1, and ft-1 (introgressed into Columbia), showed approximately $30 \%-40 \%$ reduction of AGL20 expression, but $F L C$ expression was similar to Columbia wild type (Fig. 5F). In summary, the results show that AGL20 expression is positively regulated by floweringtime genes, although it is more strongly dependent on genes in the autonomous pathway.

Similar results were obtained from late-flowering mutants in Ler, which has a weak FLC allele (Fig. 5G). All of the late-flowering mutants in Ler showed decreased levels of AGL2O expression. However, the photoperiod pathway mutants, $c 0-2, g i-3$, and $f t-1$, showed less reduction in AGL20 expression than the autonomous pathway mutants, $f c a-1$, fve-1, and fpa-1, confirming that AGL2O expression is more strongly dependent on the autonomous pathway than the photoperiod pathway also in the Ler background (Fig. 5G). Interestingly, autonomous pathway mutant fpa-1 consistently showed higher expression of $A G L 20$ than $f_{c a-1}$ and $f_{V e}-1$. It may indicate that FPA acts more or less differently with the other genes involved in the autonomous pathway. In conclusion, AGL2O expression is regulated by three floral inductive pathways, including the photoperiod, vernalization, and autonomous pathways. 


\section{Discussion}

\section{AGL20 is a floral activator}

Winter-annual Arabidopsis strains such as Stockholm and SF2 flower very late in the absence of vernalization treatment. Previous genetic analyses showed that such lateness of flowering is mainly caused by the synergistic interaction of two genes, FRI and FLC (Napp-Zinn 1985; Burn et al. 1993; Lee et al. 1993; Clarke and Dean 1994; Koornneef et al. 1994; Lee et al. 1994b). To define the regulatory mechanism of FRI and FLC, a screen for downstream factor(s) repressed by FRI and FLC was pursued by the random overexpression strategy of activation tagging mutagenesis. The rationale is that if the lateflowering phenotype of the FRI FLC line is suppressed by overexpression of a certain gene, that gene may be a downstream target gene of FRI FLC repression. One of the suppressor mutants, ag120-101D FRI FLC, shows overexpression of a MADS-domain gene, AGL20. The results presented in this study show that AGL20 acts as a floral activator and is repressed by the interaction of FRI and FLC. A positive role for AGL20 is confirmed by ag120 loss-of-function mutants, which flower late.

The expression level of AGL20 correlates very well with flowering time. In the FRI FLC lines, which flower very late, $A G L 20$ expression is very weak. The homozygous ag120-101D FRI FLC mutant overexpresses AGL20 in the FRI FLC background and flowers even earlier than Columbia. Consistently, 35S::AGL20 transformants in the FRI FLC background show accelerated flowering with increased level of AGL20 expression (Fig. 5E). Finally, fri (FN235) FLC or FRI flc-3 strains, whose flowering times are similar to that of Columbia, show similar levels of AGL20 expression as Columbia. Such a correlation between AGL20 expression level and flowering time may explain the responsiveness of ag120-101D FRI $F L C$ to both photoperiod and vernalization. The activation of endogenous AGL20 by long days and vernalization may have an additive effect on flowering of agl20101D FRI FLC.

AGL20 is very similar to the SaMADS A from Sinapsis alba (95.3\% amino acid sequence identity; Menzel et al. 1996). SaMADS A is expressed most highly at the shoot apical meristem of plants after they have been induced to flower. Based on the expression pattern, it has been suggested that SaMADS A has an important function during the transition to flowering. Our genetic analysis of AGL20 in Arabidopsis has shown that AGL20 is indeed an important regulator of flowering. In addition, we have shown that $A G L 20$ is expressed in vegetative tissues, consistent with a more general role of AGL20 in regulating phase transitions. AGL20 expression is gradually increased during vegetative growth. Such a gradual increase before flower initiation has also been observed in $L F Y$ and FT, two regulators of flowering (Blázquez et al. 1997; Kardailsky et al. 1999; Kobayashi et al. 1999).

AGL20 is a member of the MADS-domain family, a large family of transcription factors. Many MADS-domain proteins are involved in flower development. For example, AP1 and CAULIFLOWER function as floral meristem identity genes, and APETALA3 and PISTILLATA are involved in floral organ development (Riechmann and Meyerowitz 1997). However, AGL15 plays a role during embryogenesis and AGL12, AGL14, and $A G L 17$ are expressed only in root tissues, suggesting that MADS-domain family members play diverse roles in plant development (Heck et al. 1995; Rounsley et al. 1995). Interestingly, the floral repressor FLC is also a member of MADS-domain family and shows the second highest amino acid sequence identity with AGL20 among Arabidopsis MADS-domain proteins.

\section{AGL20 activates phase transition}

The interaction of FRI and FLC causes not only a delay of flowering but also a delay of phase transition during all stages of plant development. Such an overall delay in plant development is observed in most late-flowering mutants (Martínez-Zapater et al. 1995; Telfer et al. 1997). ag120-101D accelerates both the transition from the juvenile to adult phase and that from vegetative to reproductive phase in the FRI FLC background. A weaker acceleration is also found in the transition from cauline leaves with secondary shoots to flowers. The different acceleration of phase transitions may result from a dual role of $F R I$ and $F L C$, which are likely to regulate flowering not only through AGL20, but also through meristem identity genes such as $L F Y$. We propose that the interaction of FRI and FLC not only inhibits expression of $A G L 20$, but also has an independent effect on $L F Y$, as suggested by our analysis of $L F Y$ promoter activity in ag120-101D FRI FLC plants. LFY is a key regulator of flower formation, but not of vegetative phase change (Weigel and Nilsson 1995). Thus, the repression of LFY by FRI FLC may weaken the acceleration of the transition from cauline leaves with secondary shoots to flowers in ag120-101D FRI FLC. Consistent with this hypothesis, when $35 S:: L F Y$ is introduced into FRI FLC, all of the secondary shoots are converted to flowers (Nilsson et al. 1998). Although not apparent in the FRI FLC background, ag120-101Dseems to activate $L F Y$ expression once FRI FLC repression is removed. In the ag120-101D fri FLC or ag120-101D FRI flc lines, ag120-101D causes the replacement of secondary shoots by ectopic flowers as is observed in 35S::LFY. The phenotype of ag120-101D FRI FLC also supports the idea that Arabidopsis has a distinct inflorescence phase I, during which cauline leaves with associated secondary shoots are produced (Ratcliffe et al. 1998, 1999). Although homozygous ag120-101D FRI FLC lines make the transition to flowering more quickly than Columbia, these lines produce a higher number of cauline leaves than Columbia.

\section{Floral induction pathways merge to activate AGL20}

In addition to FRI and FLC, we have identified several other regulators of AGL20 expression, and a model of how three floral inductive pathways are integrated by 
AGL20 is presented in Figure 6. In this model, AGL20 plays a pivotal role in the control of flowering time, with the three floral inductive pathways merging to activate AGL20. Activation of AGL20 by the autonomous pathway genes, $L D, F C A$, and $F V E$, is likely mediated by repression of $F L C$, as is activation of AGL20 upon vernalization.

In addition to the autonomous and the vernalization pathways, the photoperiodic pathway activates AGL20. However, the reduction of AGL20 expression in $C O$, gi, and $f t$ mutants is relatively modest, even though at least $c o$ and gi mutants flower much later in long days than does ag120. These observations indicate that AGL20 is not the only factor downstream from the photoperiodic pathway. We have recently found that the photoperiod pathway and the autonomous pathway interact through AGL20 activity. ag120-101D can partly suppress the lateflowering phenotype of the co mutant $(H$. Lee and I. Lee, unpubl.), consistent with a role of AGL20 downstream from CO. Conversely, 35S::CO can compensate for the delay in flowering in $f c a$ mutants (Piñeiro and Coupland 1998). Thus, there must be some cross-talk between the autonomous and photoperiod pathway, and we propose that AGL20 is a component of this cross-talk.

A question remains which other genes integrate flowering signals. Two other candidates for integration of floral inductive signals are FT and $L F Y$. Similar to AGL20, $F T$ and $L F Y$ act partially downstream from $C O$, a gene that promotes flowering in response to long days (Simon et al. 1996; Kardailsky et al. 1999; Kobayashi et al. 1999). In addition, the expression of FT and $L F Y$ is regulated by FCA, which is involved in the autonomous pathway (Nilsson et al. 1998; J.H. Ahn and D. Weigel, pers. comm.). The analysis of ag120-101D mutants suggests

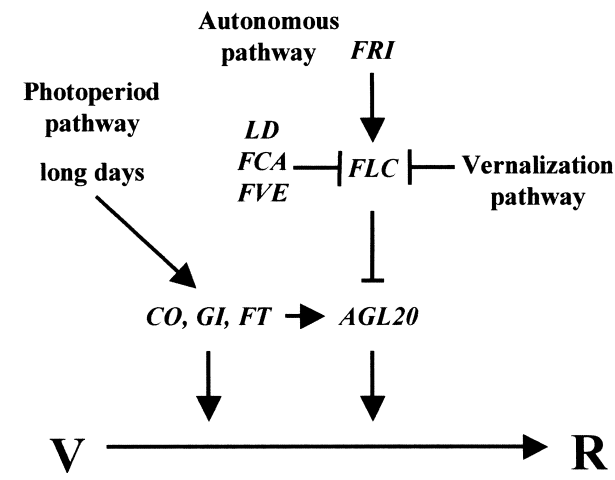

Figure 6. Model for the integrative role of AGL2O and the interaction of flowering pathways. The horizontal line represents the vegetative $(\mathrm{V})$ to reproductive $(\mathrm{R})$ transition. Arrows indicate promotion, and T-bars indicate repression. In the autonomous pathway, FRI activates FLC, and FLC represses AGL20. $A G L 20$ acts as a floral activator. Other autonomous pathway genes, such as $L D, F C A$, and $F V E$, promote flowering by activating $A G L 20$ through the repression of $F L C$. Vernalization also promotes flowering by activating AGL2O expression through the repression of $F L C$. Photoperiod pathway genes, $C O$ and GI, promote flowering by activating AGL2O but act also through other factor(s). that $L F Y$ acts at least in part downstream from AGL20. On the other hand, our data suggest that FT affects AGL20 expression, indicating that there is substantial cross-regulation among the three genes.

In addition to the photoperiod, vernalization, and autonomous pathways, it has been suggested that a gibberellin pathway promotes flowering (Levy and Dean 1998). A gibberellin biosynthetic mutant, ga1, does not flower under short days, and exogenous treatments of gibberellin accelerate flowering of many late-flowering mutants of both photoperiod and autonomous pathways (Wilson et al. 1992; Chandler and Dean 1994). The effect of gibberellin on AGL20 expression may lead to further understanding how the pathways controlling flowering in Arabidopsis are integrated.

\section{Materials and methods}

\section{Plant materials and growth conditions}

Plants (Arabidopsis thaliana) were grown in long days (16 h light $/ 8 \mathrm{~h}$ dark) or short days $(8 \mathrm{~h}$ light $/ 16 \mathrm{~h}$ dark), under cool white fluorescent lights $\left(100 \mu \mathrm{mole} / \mathrm{m}^{2} / \mathrm{sec}\right)$, at $23 \pm 2{ }^{\circ} \mathrm{C}$, $60 \pm 10 \%$ relative humidity. For vernalization studies, seeds were imbibed on $0.8 \%$ phytoagar containing half strength of Murashige-Skoog (MS) medium (GIBCO BRL) and incubated for 4 weeks at $4^{\circ} \mathrm{C}$ under short days. FRI FLC line is a Columbia near-isogenic line with FRI allele from SF2 obtained by eight backcrosses into Columbia, which was described previously (Michaels and Amasino 1999). The mutants, fri (FN235) FLC and $F R I$ flc-3, are the lines obtained by fast neutron mutagenesis of FRI FLC. The late-flowering mutants, co-1, gi-1, fve-3, ld-1, and ag120, are in the Columbia background, but co-2, gi-3, ft-1, $f d-1, f h a-1, f e-1, f w a-2, f c a-1, f v e-1$, and fpa-1 are in the Ler background. $f t-1$ introgressed into Columbia was provided by $\mathrm{D}$. Weigel (Salk Institute).

\section{Screening of activation-tagging mutants}

FRI FLC line was transformed with pSKI015 (Weigel et al. 2000) by vacuum infiltration (Bechtold et al. 1993). For screening of activation-tagging mutants, T0 seeds were sown on soil under long days and treated with $0.1 \%$ basta (AgrEvo, USA) after $7 \mathrm{~d}$. For basta segregation analysis, seeds were germinated on halfstrength MS media with $0.007 \%$ basta.

\section{Construction of 35S::AGL20}

To generate a 35S::AGL20 construct, an AGL20 cDNA was amplified by RT-PCR using total RNA extracted from Columbia leaf tissues. BamH1 sites were introduced before the ATG start codon and after the TGA stop codon in the cDNA. The primers used in the PCR were 5 '-CCC GGA TCC ATG GTG AGG GGC AAA ACT CAG-3' and 5'-CCC GGA TCC TCA CTT TCT TGA AGA ACA AGG-3'. The RT-PCR product was subcloned into the pGEM-Teasy vector (Promega) to yield pHR2. The sequence of AGL2O cDNA in pHR2 was confirmed using Sequenase version 2.0 kit (U.S. Biochemical Corp.). The 645-bp insert of pHR2 was digested with BamH1 and introduced into the BamH1 site of pCGN18 vector (Jack et al. 1994). Constructs were introduced into the FRI FLC line by vacuum infiltration.

Phylogenetic analysis

Nucleotide and predicted amino acid sequences of MADS-domain proteins in Arabidopsis were obtained from GenBank. 
Distance matrices were calculated using the DNADIST program of PHYLIP version 3.5 (Department of Genetics, University of Washington, Seattle, WA), and the numbers of nucleotide substitutions were estimated using the two-parameter method of Kimura (1980). Distance trees were constructed using the neighbor-joining method (Saitou and Nei 1987) and implemented using the NEIGHBOR program in PHYLIP.

\section{RT-PCR}

Total RNA was extracted as described previously (Puissant and Houdebine 1990). One $\mu \mathrm{g}$ of total RNA from each tissue was reverse-transcribed with oligo-d $\mathrm{T}_{12-18}$ (GIBCO BRL) in a $20 \mu \mathrm{L}$ reaction mixture using the Reverse Transcription System (Promega). After heat inactivation of the reaction mixture, PCR was performed using $1 \mu \mathrm{L}$ of the first-stranded cDNA sample with 25 pmole of the primers in a $50 \mu \mathrm{L}$ reaction. PCR conditions were as follows: $94^{\circ} \mathrm{C}(3 \mathrm{~min}) ; 25$ cycles of $94^{\circ} \mathrm{C}(30 \mathrm{sec}), 55^{\circ} \mathrm{C}(1$ $\mathrm{min}), 72^{\circ} \mathrm{C}(1 \mathrm{~min})$, and $72^{\circ} \mathrm{C}(10 \mathrm{~min})$. PCR products were electrophoresed on an agarose gel, blotted onto a NYTRAN-PLUS membrane (Schleicher \& Schuell), and hybridized with the appropriate probes. RT-PCR was repeated at least three times for the samples harvested separately. The primers used for RT-PCR were as follows: For $\beta$-tubulin (TUB2), two primers, $5^{\prime}$-CTC AAG AGG TTC TCA GCA GTA-3' and 5'-TCA CCT TCT TCA TCC GCA GTT-3' were used. For AGL20, two primers, 5'-CCC CAT ATG GTG AGG GGC AAA ACT C-3' and 5'CCC GGA TCC TCA CTT TCT TGA AGA ACA AGG-3' were used. For $F L C$, two primers, 5' -CCC CAT ATG GGA AGA AAA AAA CTA G-3' and 5'-CCC GGA TCC CTA ATT AAG TAG TGG GAG-3' were used. For AP1, two primers, 5'-GCA CCT GAG TCC GAC GTC-3' and 5'-GCG GCG AAG CAG CCA AGG-3' were used.

\section{GUS staining}

For histochemical analysis of GUS, X-gluc staining and tissue fixation were performed as described by Blázquez et al. (1997). Eight- $\mu \mathrm{m}$ sections were prepared and mounted and, after removal of paraplast with xylenes, photographed under bright field on an Olympus BX50 microscope. LFY::GUS used in this study was strain DW150-209 (Blázquez et al. 1997).

\section{Genotyping of FRI}

To identify $f_{s u 1-1 D}$ with the fri-Col allele, genomic DNA was amplified by PCR using two primers, 5'-CAA CGA CCA AAC ACA ACG AC-3' and 5'-CGC GAG ACT GAA CCT CAC GG$3^{\prime}$ and digested with Rsa1. The FRI-SF2 allele yielded four fragments $(100,200,300$, and $350 \mathrm{bp})$, and the $300 \mathrm{bp}$ fragment was replaced by a $330 \mathrm{bp}$ fragment in the fri-Col allele.

\section{Acknowledgments}

We thank S. Michaels and R. Amasino for providing fri (FN235) $F L C, F R I f l c-3$, and $f_{v e}-3$ seeds and the information for primers for FRI genotyping. We also thank D. Weigel for providing the pSKI015 vector and $f t-1$ in Columbia seeds and G. Ditta and M. Yanofsky for agl20 mutant seeds and in situ hybridization data for AGL20. Special thanks go to R. Amasino and D. Weigel for their encouragement of this study and critical reading of the manuscript. This study was supported by the Academic Research Fund (GE 1998-019-D00134) of the Korea Research Foundation. H. Lee, E. Park, and E. Cho are supported by Brain Korea 21 program.
The publication costs of this article were defrayed in part by payment of page charges. This article must therefore be hereby marked "advertisement" in accordance with 18 USC section 1734 solely to indicate this fact.

\section{References}

Bechtold, N., Ellis, J., and Pelletier, G. 1993. In planta Agrobacterium-mediated gene transfer by infiltration of adult Arabidopsis thaliana plants. C. R. Acad. Sci. 316: 1194-1199.

Blázquez, M., Soowal, L., Lee, I., and Weigel, D. 1997. LEAFY expression and flower initiation in Arabidopsis. Development 124: 3835-3844.

Blázquez, M.A., Green, R., Nilsson, O., Sussman, M.R., and Weigel, D. 1998. Gibberellins promote flowering of Arabidopsis by activating the $L E A F Y$ promoter. Plant Cell 10: 791-800.

Burn, J.B., Smyth, D.R., Peacock, W.J., and Dennis, E.S. 1993. Genes conferring late flowering in Arabidopsis thaliana. Genetica 90: 147-155.

Chandler, J. and Dean, C. 1994. Factors influencing the vernalization response and flowering time of late flowering mutants of Arabidopsis thaliana (L.) Heynh. I. Exp. Bot. 45: $1279-1288$.

Clarke, J.H. and Dean, C. 1994. Mapping FRI, a locus controlling flowering time and vernalization response in Arabidopsis thaliana. Mol. Gen. Genet. 242: 81-89.

Fowler, S., Lee, K., Onouchi, H., Samach, A., Richardson, K., Morris, B., Coupland, G., and Putterill, J. 1999. GIGANTEA: A circadian clock-controlled gene that regulates photoperiodic flowering in Arabidopsis and encodes a protein with several possible membrane-spanning domains. EMBO $T$. 18: 4679-4688.

Guo, H., Yang, H., Mockler, T.C., and Lin, C. 1998. Regulation of flowering time by Arabidopsis photoreceptors. Science 279: 1360-1363.

Hayashi, H., Czaja, I., Lubenow, H., Schell, J., and Walden, R. 1992. Activation of a plant gene by T-DNA tagging auxinindependent growth in vitro. Science 258: 1350-1352.

Heck, G.R., Perry, S.E., Nichols, K.W., and Fernandez, D.E. 1995. AGL15, a MADS domain protein expressed in developing embryos. Plant Cell 7: 1271-1282.

Jack, T., Fox, G.L., and Meyerowitz, E.M. 1994. Arabidopsis homeotic gene APETALA3 ectopic expression: Transcriptional and posttranscriptional regulation determine organ identity. Cell 76: 703-716.

Kardailsky, I., Shukla, V.K., Ahn, J.H., Dagenais, N., Christensen, S.K., Nguyen, J.T., Chory, J., Harrison, M.J., and Weigel, D. 1999. Activation tagging of the floral inducer FT. Science 286: 1962-1965.

Kimura, M. 1980. A simple method for estimating evolutionary rates of base substitutions through comparative studies of nucleotide sequences. J. Mol. Evol. 16: 111-120.

Kobayashi, Y., Kaya, H., Goto, K., Iwabuchi, M., and Araki, T. 1999. A pair of related genes with antagonistic roles in mediating flowering signals. Science 286: 1960-1962.

Koornneef, M., Blankestijn-de Vries, H., Hanhart, C., Soppe, W., and Peeters, T. 1994. The phenotype of some late-flowering mutants is enhanced by a locus on chromosome 5 that is not effective in the Landsberg erecta phenotype. Plant J. 6: 911919.

Koornneef, M., Alonso-Blanco, C., Peeters, A.J., and Soppe, W. 1998. Genetic control of flowering time in Arabidopsis. Annu. Rev. Plant Physiol. Plant Mol. Biol. 49: 345-370.

Lee, I. and Amasino, R.M. 1995. Effect of vernalization, photo- 
period, and light quality on the flowering phenotype of Arabidopsis plants containing the FRIGIDA gene. Plant Physiol. 108: 157-162.

Lee, I., Bleecker, A., and Amasino, R.M. 1993. Analysis of naturally occurring late flowering in Arabidopsis thaliana. Mol. Gen. Genet. 237: 171-176.

Lee, I., Aukerman, M.J., Gore, S.L., Lohman, K.N., Michaels, S.D., Weaver, L.M., John, M.C., Feldmann, K.A., and Amasino, R.M. 1994a. Isolation of LUMINIDEPENDENS: A gene involved in the control of flowering time in Arabidopsis. Plant Cell 6: 75-83.

Lee, I., Michaels, S.D., Masshardt, A.S., and Amasino, R.M. 1994b. The late-flowering phenotype of FRIGIDA and mutations in LUMINIDEPENDENS is suppressed in the Landsberg erecta strain of Arabidopsis. Plant J. 6: 903-909.

Levy, Y.Y. and Dean, C. 1998. The transition to flowering. Plant Cell 10: 1973-1989.

Macknight, R., Bancroft, I., Lister, C., Page, T., Love, K., Schmidt, R., Westphal, L., Murphy, G., Sherson, S., Cobbett, C., et al. 1997. FCA, a gene controlling flowering time in Arabidopsis, encodes a protein containing RNA-binding domains. Cell 89: 737-745.

Mandel, M.A. and Yanofsky, M.F. 1995. A gene triggering flower development in Arabidopsis. Nature 377: 522-524.

Martínez-Zapater, J.M., Jarillo, J.A., Cruz-Alvarez, M., Roldán, M., and Salinas, J. 1995. Arabidopsis late-flowering fve mutants are affected in both vegetative and reproductive development. Plant J. 7: 543-551.

Menzel, G., Apel, K., and Melzer, S. 1996. Identification of two MADS box genes that are expressed in the apical meristem of the long-day plant Sinapis alba in transition to flowering. Plant J. 9: 399-408.

Michaels, S.D. and Amasino, R.M. 1999. FLOWERING LOCUS $C$ encodes a novel MADS domain protein that acts as a repressor of flowering. Plant Cell 11: 949-956.

Napp-Zinn, K. 1985. Arabidopsis thaliana. In CRC Handbook of Flowering (ed. H.A. Halevy), pp. 492-503. CRC Press, Boca Raton, FL.

Nilsson, O., Lee, I., Blázquez, M.A., and Weigel, D. 1998. Flowering-time genes modulate the response to $L E A F Y$ activity. Genetics 150: 403-410.

Park, D.H., Somers, D.E., Kim, Y.S., Choy, Y.H., Lim, H.K., Soh, M.S., Kim, H.J., Kay, S.A., and Nam, H.G. 1999. Control of circadian rhythms and photoperiodic flowering by the Arabidopsis GIGANTEA gene. Science 285: 1579-1582.

Poethig, R.S. 1990. Phase changes and the regulation of shoot morphogenesis in plants. Science 250: 923-930.

Piñeiro, M. and Coupland, G. 1998. The control of flowering times and floral identity in Arabidopsis. Plant Physiol. 117: $1-8$.

Puissant, C. and Houdebine, L.M. 1990. An improvement of the single-step method of RNA isolation by acid guanidinium thiocyanate phenol-chloroform extraction. Biotechniques 8: 148-149.

Putterill, J., Robson, F., Lee, K., Simon, R., and Coupland, G. 1995. The CONSTANS gene of Arabidopsis promotes flowering and encodes a protein showing similarities to zinc finger transcription factors. Cell 80: 847-857.

Ratcliffe, O.J., Amaya, I., Vincent, C.A., Rothstein, S., Carpenter, R., Coen, E.S., and Bradley, D.J. 1998. A common mechanism controls the life cycle and architecture of plants. Development 125: 1609-1615.

Ratcliffe, O.J., Bradley, D.J., and Coen, E.S. 1999. Separation of shoot and floral identity in Arabidopsis. Development 126: $1109-1120$.

Riechmann, J.L. and Meyerowitz, E.M. 1997. Determination of floral organ identity by Arabidopsis MADS domain homeotic proteins AP1, AP3, PI, and AG is independent of their DNA-binding specificity. Mol. Biol. Cell 8: 1243-1259.

Rounsley, S.D., Ditta, G.S., and Yanofsky, M.F. 1995. Diverse roles for MADS box genes in Arabidopsis development. Plant Cell 7: 1259-1269.

Ruiz-García, L., Madueño, F., Wilkinson, M., Haughn, G., Salinas, J., and Martínez-Zapater, J.M. 1997. Different roles of flowering-time genes in the activation of floral initiation genes in Arabidopsis. Plant Cell 9: 1921-1934.

Saitou, N. and Nei, M. 1987. The neighbor-joining method: A new method for reconstructing phylogenetic trees. Mol. Biol. Evol. 4: 406-425.

Sanda, S.L. and Amasino, R.M. 1995. Genetic and physiological analysis of flowering time in the $\mathrm{C} 24$ line of Arabidopsis thaliana. Weeds World 2: 2-8.

Schultz, E.A. and Haughn, G.W. 1993. Genetic analysis of the floral initiation process (FLIP) in Arabidopsis. Development 119: $745-765$.

Sheldon, C.C., Burn, J.E., Perez, P.P., Metzger, J., Edwards, J.A., Peacock, W.J., and Dennis, E.S. 1999. The FLF MADS box gene: A repressor of flowering in Arabidopsis regulated by vernalization and methylation. Plant Cell 11: 445-458.

Sheldon, C.C., Rouse, D.T., Finnegan, E.J., Peacock, W.J., and Dennis, E.S. 2000. The molecular basis of vernalization: The central role of FLOWERING LOCUS C (FLC). Proc. Natl. Acad. Sci. 97: 3753-3758.

Simon, R., Igeño, M.I., and Coupland, G. 1996. Activation of floral meristem identity genes in Arabidopsis. Nature 382: 59-62.

Telfer, A., Bollman, K.M., and Poethig, R.S. 1997. Phase change and the regulation of trichome distribution in Arabidopsis thaliana. Development 124: 637-644.

Weigel, D. 1995. The genetics of flower development: From floral induction to ovule morphogenesis. Annu. Rev. Genet. 29: 19-39.

Weigel, D. and Nilsson, O. 1995. A developmental switch sufficient for flower initiation in diverse plants. Nature 377: 495-500.

Weigel, D., Ahn, J.H., Blazquez, M.A., Borevitz, J.O., Christensen, S.K., Fankhauser, C., Ferrandiz, C., Kardailsky, I., Malanchauvil, E.J., Neff, M.M., et al. 2000. Activation tagging in Arabidopsis. Plant Physiol. 122: 1003-1013.

Wilson, R.N., Heckman, J.W., and Somerville, C.R. 1992. Gibberellin is required for flowering in Arabidopsis thaliana under short days. Plant Physiol. 100: 403-408. 


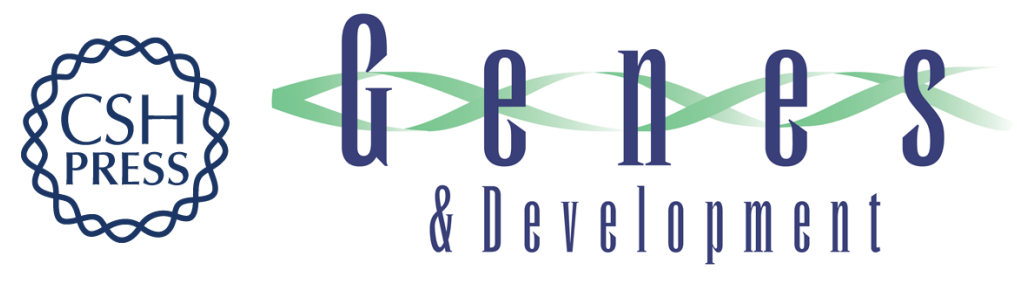

\section{The AGAMOUS-LIKE 20 MADS domain protein integrates floral inductive pathways in Arabidopsis}

Horim Lee, Sung-Suk Suh, Eunsook Park, et al.

Genes Dev. 2000, 14:

Access the most recent version at doi:10.1101/gad.813600

References This article cites 47 articles, 27 of which can be accessed free at: http://genesdev.cshlp.org/content/14/18/2366.full.html\#ref-list-1

License

Email Alerting

Receive free email alerts when new articles cite this article - sign up in the box at the top Service right corner of the article or click here.

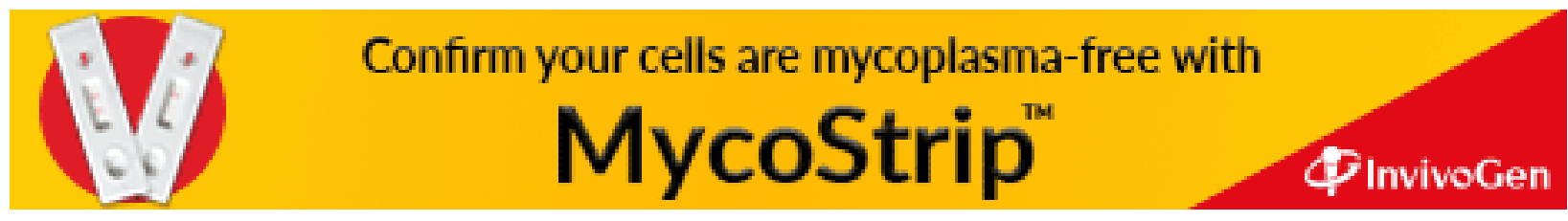

